\title{
A Source-Based Multicast Scheme in IEEE 802.16 Mesh Mode
}

\author{
Mortaza Maleki ${ }^{\text {a1 }}$,Saleh Yousefi ${ }^{\text {b2 }}$,Ali Azarpeyvand ${ }^{\text {a } 3}$ \\ ${ }^{a}$ Department of Computer Engineering Islamic Azad University, Zanjan Branch Zanjan, Iran \\ ${ }^{b}$ Department of Computer Engineering Urmia University Urmia, Iran
}

\begin{abstract}
WiMAX based on IEEE 802.16 is known as one of effective technologies for transmission of multimedia to groups of receivers. There are two operational modes in WiMAX: point to multi point (PMP) for single hop transmission and a mesh node for multi-hop transmission. The current standard only defines unicast transmission under mesh mode thus the performance is deteriorated for multimedia applications with multicast nature. In this paper, we first propose a mechanism for source-based tree topology construction aiming at facilitating multicast transmission in WiMAX mesh network. In the proposed approach, the multicast tree is constructed considering available link's bandwidth and multicast group members. After constructing the multicast tree we propose a novel scheduling algorithm to improve the transmission throughout. In the proposed scheduling algorithm a parent node uses broadcasting when the number of its children belonging to a multicast group excesses a threshold. Extensive simulation results show that the proposed multicast mechanism improves multicast transmission efficiency and reduces scheduling time slots noticeably in comparison to the approach mandated by the IEEE 802.16 standard.
\end{abstract}

Index Terms: IEEE 802.16; WiMAX; Mesh mode; Multicast

(C) 2012 Published by MECS Publisher. Selection and/or peer review under responsibility of the Research Association of Modern Education and Computer Science

\section{INTRODUCTION}

WiMAX based on IEEE 802.16 is one of the effective and promising technologies for implementation wireless network that is interested by industrial and academic survey. Two transmission modes are defined in the IEEE 802.16 including PMP and Mesh mode. PMP mode is defined for one hop rang, however the Mesh mode is designed for supporting multi hop communication. The standard [1] only defines the multicast connection in PMP mode in one hop range, and the connection ID(CID) used for the Multicast service is the same for all subscribe stations(SSs) that participate in the multicast, so that one unit multicast data can be transmitted in one frame to all destination SSs that join the multicast session. The standard only defines unicast transmission under Mesh mode, but doesn't specify multicast transmission scheme. For transmission of packet to members that belongs to same group and are located to different geographical region: one approach is for the source to use replicated unicast [2], that is, the source created a separate packet containing identical data for

\footnotetext{
* Corresponding author.

E-mail address: ${ }^{1}$ Mortaza.maleki @ gmail.com, ${ }^{2}$ syousefi@urmia.ac.ir, ${ }^{3}$ azarpeyvand @ gmail.com
} 
each destination host in the group. However, this approach increase burden on the source. Another possible approach to multicasting is to broadcast the data [2]. This removes the burden from the source but extend the burden to the other host in the network.

To dominate the stated restrictions, is to use multicast concept that data packet is transmitted to group members. The basic approach for multicasting is to establish a multicast tree among group members [3]. In this paper, we propose a scheme to construct multicast tree among group members and the source of packet generator, however in the constructed tree, some nodes use unicast transmission and others under special condition use broadcast transmission. This scheme effectively reduces needed time slots to packet transmission. We use effective and Varity parameters in wireless network to construct tree as propagation delay and bandwidth between two nodes and hop count to source.

The rest of this paper organized as following: in section II, The Control Message in WiMAX Mesh mode is briefly discussed. In section III, The problem of multicast is mentioned. In section IV, Related work about multicast is evaluated. In section V, the proposed scheme is given with a case study. In section VI, we evaluate the proposed scheme. In section VII, we summaries our conclusions. Finally, the future works are stated.

\section{Control Message in WiMAX Mesh mode}

In centralized scheduling, Mesh-CSCF (centralized scheduling configuration) Message carries the information of channel configuration and routing tree information, while Mesh- $\mathrm{CSCH}$ (centralized scheduling) message carries the information of bandwidth request and grant. The BS generates grant messages and broadcast it to all its neighbors, and all the BS neighbors rebroadcast this message. All SSs transmit resource request through Mesh-CSCH: Request. The transmission order is determined by the hop count and ID of each node. Mesh-NENT (network entry) message provides the means for a new node to gain Synchronization and initial network entry. IGMP message is used for management of multicast group member into network and prune of the tree structure. In the other words, IGMP is always the language spoken between hosts and routers.

\section{THE PROBLEM}

Multicasting is the process of sending data to a group of receivers. It might be argued that unicasting and broadcasting are subsets of multicasting. In the case of unicasting, there is only a single member of the group; in the case of broadcasting, all possible receivers are members of the group. This section demonstrates why such an argument is valid only on a conceptual level; in networking, at least, distinct differences exist between multicasting, unicasting, and broadcasting.

Suppose one of the hosts is a source (S) of data that must be delivered to a group $(\mathrm{G})$ of receivers. There is more than one receiver, but the group does not contain all possible receivers. One approach is for the source to use a replicated unicast [2]. That is, the source creates a separate packet containing identical data for each destination host in the group. Each packet is then unicast to a specific host. If there are only a few destinations, this scheme works fine. In fact, many multicast applications in use today actually utilize replicated unicast. As the number of recipients grows into the hundreds or thousands, however, the burden on the host to create and send so many copies of the same data also increases. There are also problems if the data is delay-sensitive. If all the copies of packet number 2 must wait for all the copies of packet number 1 to be queued and sent, the queuing delay can introduce unacceptable gaps in the data stream.

Another possible approach to multicasting is to broadcast the data [2]. This removes the burden from the source and its local facilities, which now have to send only a single copy of each packet, but it can extend the burden to the other hosts in the network. Each host must accept a copy of the broadcasted packet and process the packet. It is only at the higher layers, or possibly within the application itself, that disinterested hosts recognize that the packet is to be discarded. If the number of hosts in the receiving group is small in relation to the total number of hosts in the network, this processing burden can again be unacceptable. 
Multicasting allows the source to send a single packet to a single multicast destination address, thus removing the processing burden of replicating packets. Any receiver that is listening for the multicast address can receive the packet, removing the need for disinterested hosts to process an unwanted packet. And unlike broadcast packets, multicast-aware routers can forward multicast packets.

The basic approach for multicasting is to establish a multicast tree for a group of node that constitute the multicast session. How to construct a multicast tree in mesh mode is undefined in the standard [3].

\section{Related works}

Multicast for computer networks have been studied by many groups and several multicast schemes are proposed and classified in various ways. Algorithms that proposed for multicast routing are classified in two categories, some include multicast routing that classified in wire network as DVMRP [2], MOSPF [2], PIM [2] and CBT [2] versus wireless as MAODV [4]. Other classification exists based on link scheduling: In [5] the frame is proposed for downlink scheduling with multimedia traffic, in [6] a cooperative multicast scheduling scheme is proposed for IPTV service over IEEE 802.16 networks, which used two phase cooperative transmission to exploit the spatial diversity gain among nodes. The other classification is related to multicast mechanisms: in [3] the mechanism is proposed based on tree that generated traffic in source is transmitted to rendezvous point as BS, then a tree is constructed with the root to BS and traffic through this tree is transmitted to group members. In [7] is proposed an efficient MAC layer multicast approach that use a reserved field of MCA-REQ message for mapping the multicast address to CID (connection ID). We propose our own views of classification of multicast schemes that tree are based on rendezvous point and source. In the former, the traffic is sent to rendezvous point then is transmitted to group members and in the later, the tree is rooted from source and traffic is transmitted directly from source to group members.

\section{The proposed scheme}

\section{A. Routing Tree}

Dijkstra algorithm has used in previous schemes like [3] for construct routing tree among source and group members in which propagation delay has consider as link weight between two nodes. For construction of tree, we evaluate both propagation delay and bandwidth between two nodes. In the other words, we evaluate whether the link has sufficient bandwidth to support from new traffic. We suppose that multicast traffic of two different sources is entered to node with ID 0 in this part of network as shown in Fig 1. Now node with ID 0 must construct a tree among itself and group members. Nodes with ID 3, 5 and 6 belongs to first group, so this tree construct as Fig 2. We must construct another tree among node with ID 0 and the second group member with ID 1, 2, 7 and 8, and suppose link with ID L_1_3 does not have enough bandwidth to support the traffic of second group. With considering propagation delay between two nodes, if we use Dijkstra algorithm, link with ID L_1_3 is selected without enough bandwidth, but with our own proposed approach, an alternative link as ID L_4_7 is selected.

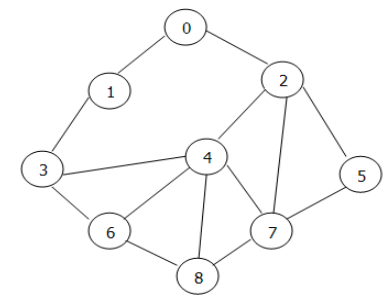

Fig.1- a part of network topology 


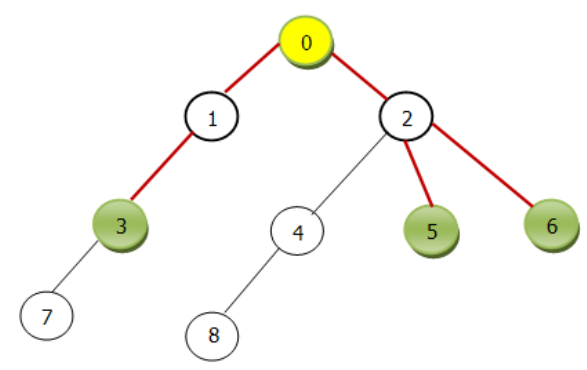

Fig. 2- Routing Tree of First Group

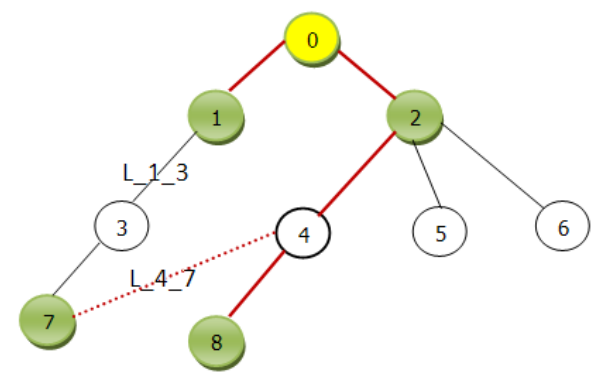

Fig 3- Routing Tree of second Group

\section{B. Multicast Tree}

In routing tree constructed among source and group members, some of the links don't eliminated among adjacent nodes, so multicast traffic is not transmitted over these links.

There are four types of nodes into the routing tree:

1- The node that packet generator.

2- The nodes that are as traffic receiver.

3- The nodes that aren't source and receiver, but are located in routing among source and receiver.

4- The nodes that aren't source and receiver and also is not located between source and receiver.

Because of multicast traffic is transmitted from source to group members, the first three categories would be in the tree, but the fourth category would prune from the tree.

We use a parameter as MEi after constructing the routing tree. MEi shows the number of receiver nodes in adjacent with node $i$. if the number of these receivers is more than threshold level, receiver nodes in adjacent with $i$ will change their father and will receive it's multicast traffic from i(i itself may be receiver or no receiver) and node i can broadcast it's traffic. If ME parameter of located nodes over the multicast tree after receiving traffic are more than threshold level, a node with broadcasting over all of its downlink interface (it's children), will deliver a multicast traffic unit to all of its children in one time slot. If a node has ME parameter less than the threshold level, a node in separate time slots will transmit the traffic in each of its downlink interfaces (to children).

\section{Multicast Tree Construction Algorithm}
Initialization
1. Get neighbor list from CSCF message.
2. Get propagation delay and bandwidth among nodes.
3. Generate Receiver node set R.
4. Initial multicast tree node set, $\mathrm{M} \leftarrow\{\mathrm{R}\} \mathrm{U}\{\mathrm{S}\}$ 
5. Initial Intermediate node set, $\mathrm{I} \leftarrow\{\varphi\}$.

6. Initial Broadcaster node set, $\mathrm{B} \leftarrow\{\varphi\}$.

7. Implement Modified Dijkstra algorithm and Construct tree.

8 . For $\mathrm{i} \leftarrow 0$ to $\mathrm{N}-1$ do $\quad / / \mathrm{N}$ is number of node.

Generate Father[i]. //father of node i.

Generate Height[i]. //hop count of node i to source.

Generate child[i]. // Childs of node i.

Generate ME[i]. /* number of receiver node in

Begin The neighbor of node $\mathrm{i} *$.

For $1 \leftarrow 1$ to $\mathrm{L}$ do //from level one to leaf?

Begin

While (node I is in level 1) do

If (ME[i] >= threshold)

Begin

While (node $\mathrm{k}$ is in neighbor of $\mathrm{i}$ and $\mathrm{k}$ is receiver)

Begin

Remove Father k from list.

Add $\mathrm{i}$ as new father of $\mathrm{k}$.

End while.

$\{\mathrm{B}\} \leftarrow\{\mathrm{B}\} \mathrm{U}\{\mathrm{i}\} . \quad / /$ add $\mathrm{i}$ to Broadcaster node set.

$\{\mathrm{M}\} \leftarrow\{\mathrm{M}\} \mathrm{U}\{\mathrm{i}\}$. //add i to multicast tree.

End for.

End if

Prune Tree ( ).

Computation Number of time slot ( ).

End.

\section{Evaluation and experimental results}

In this section, we use Fig 4 to compare proposed scheme with standard scheme (transferring multicast into multiple unicast). We also evaluate threshold parameter ME with variety numbers.

We give an example for clarifying the proposed scheme. Suppose the source with ID 9 (yellow color) transmit one

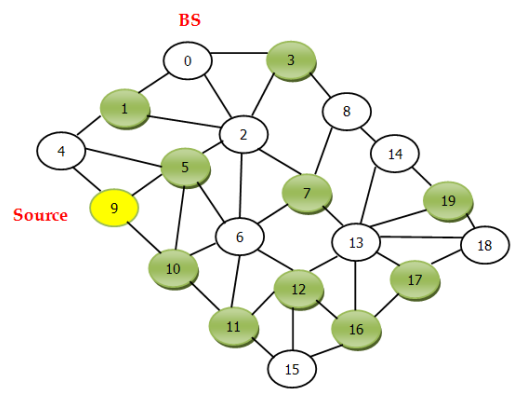

Fig.4- network Topology

data unit to group members (green color). The nodes that is white as known nodes mediator. Routing tree in

Fig 5 and multicast tree rooted from source in Fig 6 are shown. Routing tree is constructed with performing 
Dijkstra algorithm on network topology. To simplify, we suppose that value 1 is the cost of between two nodes and bandwidth is sufficient.

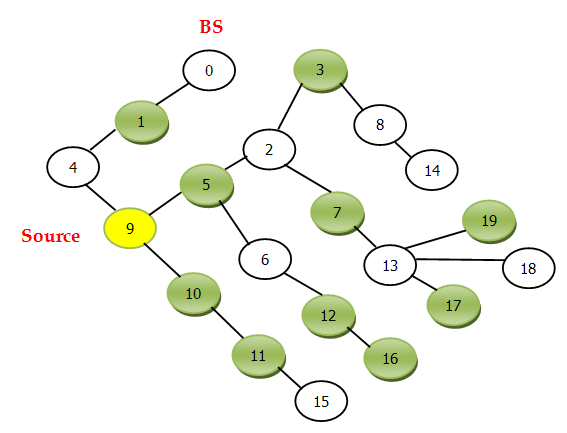

Fig. 5- Routing Tree

After constructing routing tree, according to the sequence from root to leaf, all of adjacent receivers are identified in one hop rang. If the number of receiver on one hop range are larger than or equal to threshold, so the receiver nodes will change its original father node. In this example, we suppose value 3 for threshold. As we observe, there are more than 3 receivers in lower levels or equal levels with node ID 2, so the node like ID 1 has changed its father, and then it will receive its multicast traffic from node ID 2. The broadcast action is done by node 2 because the number of receivers in adjacent are larger than or equal to threshold.

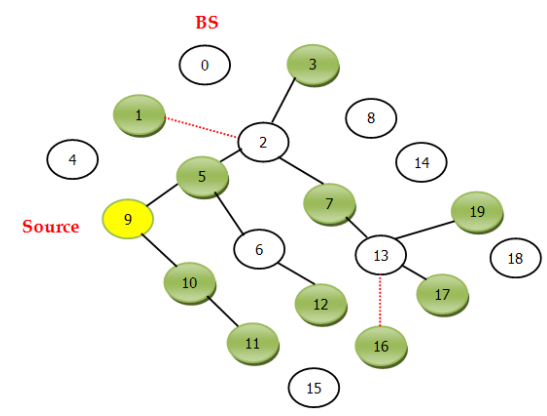

Fig. 6- Multicast Tree

In Fig 7, the transitions of one data unit from source to group members are shown by proposed scheme and standard scheme.

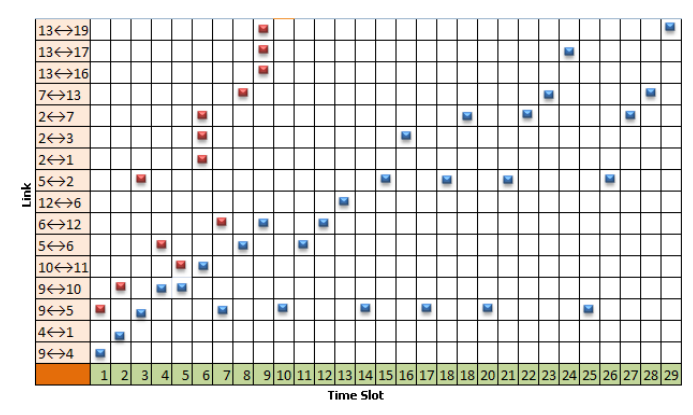

Fig. 7- Time Slot Comparison 
According to Fig 7, in standard scheme, 29 time slots are used for the transmission of one multicast data unit to group members, while only 9 time slots are needed for the transmission to all receiver nodes from source. We also observe in Fig 7 that the number of necessary time slots in standard scheme is three-fold of proposed scheme, so proposed scheme considerably reduce delay and 20 time slot are saved (68\% of 29). Fig 8 illustrates the results of 20 rounds of simulation with different number of group members and different value of thresholds. As shown on Fig 8, on the average, threshold $\mathrm{ME}=2$ need least number of time slots and on the average threshold $\mathrm{ME}=4$ need most number of time slots.

In Fig 9, the average number of broadcast was shown in terms of number of group members and different threshold.

As was observed in Fig 9, however the threshold ME=2 need averagely for the least number of time slots to transmit the multicast traffic, threshold $\mathrm{ME}=2$ has the most number of broadcast. As was shown in Fig 8 and 9 , the average of time slots at threshold $\mathrm{ME}=2$ and $\mathrm{ME}=3$ are approximately equal to node numbers 8 and 12 . At threshold $\mathrm{ME}=2$, the average of broadcast number into the network are approximately three-fold of broadcast number $\mathrm{ME}=3$.

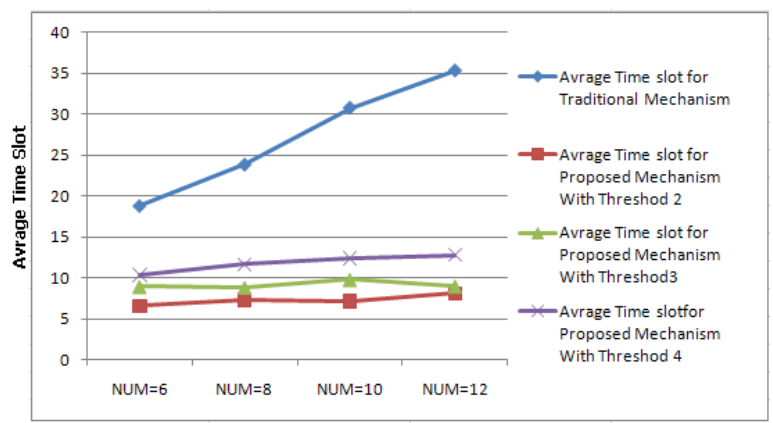

Fig. 8- Average Time Slots Comparison

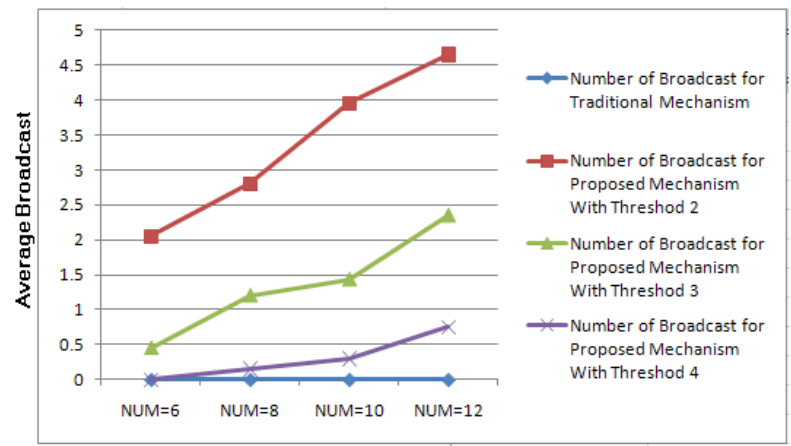

Fig. 9- Average Broadcast Compariso

\section{7. conclusions}

In this paper, we have proposed: 1) an approach for source based multicast tree construction in WiMAX mesh networks, and, 2) a novel scheduling algorithm taking advantage of the established multicast tree. In the proposed scheduling algorithm parent nodes can use one slot for transmitting data to several group members. Therefore our proposed scheduling approach outperforms legacy scheduling approaches which are based on solely unicasting. Result of simulation showed that the number of scheduling time slots is reduced noticeably. 


\section{Future Works}

As mentioned in this paper, one nodes as transmitter act in each time slot. We follow the scheme for future study by considering on interference among nodes, the transmission is possible for more than one node in a time slot, this scheme reduce considerably the number of time slot for transmitting packet to group members.

\section{References}

[1] IEEE Std 802.16-2004, Part 16: Air interface for Fixed Broadband Wireless Access Systems,1 October 2004.

[2] J.Doyle, J.DeHaven, April 11, 2001, " Routing TCP/IP”, Cisco Press.

[3] J.Chen, W.Jiao, P.Jiang, Q.Guo," A Multicast Mechanism in WiMAX Mesh Network”,IEEE 2006.

[4] Royer E, Perkins C, "Multicasting operation of the ad-hoc on-demand distance vector routing protocol", In: Proc. of MobiCom'99; 1999. p. 207-18.

[5] H.Du, J.Liu, J.Liang, "Downlink scheduling for multimedia, multicast and broadcast over mobile wimax: connection-oriented multistate adaptation", IEEE Wireless Communications, August 2009, pp.72-79.

[6] F.Hou, L.X. Cai, J.She, P.H.Ho, X. Shen, J. Zhang," Cooperative Multicast Scheduling Scheme for IPTV Service over IEEE 802.16 Networks", publication in the ICC 2008 proceedings, ,pp.2566-2570.

[7] K.S.Lee, S.W.Rhee, H.Y.Youn," A MAC Layer Multicasting Approach for WiMAX Access Networks", Sixth Annual IEEE International Conference on Pervasive Computing and Communications ,pp. 348353,2008 . 